\title{
Funciones ejecutivas y juicio moral en alumnos pertenecientes a realidades socio-culturales distintas: Lima, Ayacucho y Huancavelica
}

\section{Executive functions and moral judgment in students of different social and cultural realities: Lima, Huancavelica and Ayacucho}

\author{
Ricardo Canales ${ }^{1 *}$, Esther Velarde ${ }^{2}$, \\ Susana Lingán ${ }^{3}$ \\ Universidad Nacional Mayor de San Marcos
}

\author{
Luis Echavarría ${ }^{4}$ \\ Universidad Tecnológica del Perú
}

Recibido: $15-10-16$

Aceptado: $01-06-17$

\section{Resumen}

El estudio buscó determinar si existen diferencias en los niveles de desarrollo del juicio moral y en las funciones neuropsicológicas ejecutivas en estudiantes de tres instituciones públicas de educación superior del país, considerando su diversa realidad social y cultural.

Para examinar el Juicio Moral, se evaluó a 48 alumnos de la Universidad de San Marcos, 77 alumnos de la Universidad de Huancavelica y 84 alumnos del Instituto Pedagógico Superior de Ayacucho, con una prueba de Juicio moral (adaptación del SROM de Gibbs y Widaman), y para examinar Funciones ejecutivas, se tomó en cuenta a 23 alumnos de la Universidad de San Marcos, 20 de la Universidad de Huancavelica y 15 alumnos de la Universidad de Huamanga, examinándolos con la Batería de funciones ejecutivas BANFE (Flores- Ostrosky, 2012). Los resultados mostraron diferencias importantes en los niveles de desarrollo del juicio moral a favor del grupo de Lima, en contraste a los niveles alcanzados por los alumnos de Huancavelica. De otro lado, en la mayoría de dimensiones neuropsicológicas ejecutivas del lóbulo frontal examinadas, los puntajes favorecen igualmente al grupo de estudiantes de Lima. En conclusión se encontraron diferencias significativas entre los grupos de estudiantes examinados pertenecientes a diferentes realidades socioculturales en su desempeño en el juicio moral y las funciones neuropsicológicas ejecutivas del lóbulo frontal.

Palabras claves: Funciones neuropsicológicas ejecutivas, juicio moral, estudiantes universitarios, realidad socio-cultural.

\footnotetext{
*Autor para correspondencia

1. Facultad de Psicología, Universidad Nacional Mayor de San Marcos.

Email: rcanalesg@unmsm.edu.pe

2. Facultad de Psicología, Universidad Nacional Mayor de San Marcos.

Email: evelardec@unmsm.edu.pe

3. Facultad de Psicología, Universidad Nacional Mayor de San Marcos.

Email: ksusanalingan39@gmail.com

4. Universidad Tecnológica del Perú. Email: 1mer212@outlook.com
} 


\begin{abstract}
The study sought to determine whether there are differences in levels of development of moral judgment and executive neuropsychological functions in students from three public institutions of higher education in the country, considering its diverse social and cultural reality. To examine the Moral Judgment, 48 students from the University of San Marcos, 77 students from the University of Huancavelica and 84 students of the Higher Pedagogical Institute of Ayacucho he was evaluated with a test of moral judgment (adaptation of SROM Gibbs and Widaman) and to examine executive functions, took into account 23 students from the University of San Marcos, 20 of University of Huancavelica and 15 students from the University of Huamanga, examining the battery BANFE executive functions (Flores- Ostrosky, 2012) . The results showed significant differences in the levels of development of moral judgment in favor of the group of Lima, in contrast to the levels reached by the students of Huancavelica. On the other hand, in most dimensions executive Neuropsychological frontal lobe examined, scores also favor the group of students from Lima.

In conclusion significant differences between groups of students tested from different sociocultural realities in their performance in moral judgment and executive neuropsychological functions of the frontal lobe.
\end{abstract}

Keywords: Neuropsychological executive functions, moral judgment, university students, sociocultural reality.

La ola de violencia y criminalidad, la inseguridad ciudadana, la detención de autoridades corruptas acusada de malversación y robo, se ha vuelto pan de cada día en el país. Frente a este panorama sombrío, Neyra (1997) planteó el concepto de anomia social para dar a entender que había momentos históricos en que una sociedad entra en crisis: las instituciones básicas se tambalean, la infracción a la norma impera, hay carencia de perspectivas y liderazgo, la impunidad, el individualismo extremo y el pragmatismo se imponen.

Una pregunta principal, desde el punto de vista de la investigación, es: ¿Cómo explicar dicha crisis moral y la corrupción imperante? Las respuestas podrían venir desde distintos ángulos: histórico, social, político, económico; pero ¿qué puede aportar la psicología al respecto?

Para el presente estudio, se parte de la consideración de la diversa y compleja realidad socio- cultural en que crecen los niños y jóvenes en el Perú. González (2006) remarcó la importancia de los factores disortogénicos en el desarrollo psicológico del niño y adolescente peruano. Es decir, como la pobreza extrema, la marginalidad social, el analfabetismo de los padres, los problemas de bilinguismo quechua- castellano, etc. influyen negativamente en dicho desarrollo. De otro lado debe considerarse el problema de las familias modernas: padres que para sobrevivir tienen que multiplicarse en los trabajos pasando cada vez más tiempo fuera de casa, quedando los niños mucho tiempo solos o acompañados tan solo del televisor.

Y como trasfondo de todo ello, hay considerar que vivimos en medio de una atmósfera cultural que privilegia lo banal, lo superficial, lo efímero, y donde el criterio de éxito en la vida es solamente ganar dinero y ser famoso en la forma que sea (Vargas Llosa, 2012). 
De tal modo que quando los niños llegan a la escuela, traen ya una serie de dificultades - en cuya base hay una realidad neuropsicológica- lo cual no les permite formar y adquirir las competencias básicas, indispensables para insertarse en el mundo moderno: lectura, escritura, matemáticas, ciencias. Pero además traen serias dificultades en los procesos psíquicos complejos que tienen que ver con la planificación, anticipación, verificación, inhibición de los impulsos y regulación de la conducta, funciones que según diversos estudios, corresponde ejecutar a los lóbulos frontales (Luria, 1979; Ortiz, 2004; Flores y Ostrosky- Solis, 2008).

De otro lado, considerando los graves problemas por los que atraviesa la educación peruana manifestado en los últimos lugares alcanzados por los alumnos peruanos en lectura y comprensión lectora (PISA 2012), pareció importante considerar la participación del factor docente en dicha problemática, $\mathrm{y}$ en tal sentido era necesario acercarse al estudio de lo que estaría ocurriendo a nivel mental y neuropsicológico con los estudiantes universitarios de la carrera de educación de distintas regiones del país.

En relación a todo ello, es que se tomaron en cuenta en primer lugar, los estudios sobre el desarrollo moral realizados en nuestro medio. Montenegro (1999) examinando el Juicio Moral y Nivel de Pensamiento en 60 maestros de educación inicial, con el Test de Operaciones Formales Combinatorias de Longeot y la Escala de Reflexión Socio Moral de Gibbs y Widaman para el Juicio moral, encontró que existía relación entre un bajo nivel de pensamiento alcanzado y el nivel de desarrollo del juicio moral en la muestra estudiada. Canales (1991), efectuó un estudio sobre juicio moral y actitudes ante las drogas en adolescentes de sectores populares de Lima Metropolitana. Utilizó como instrumentos la Escala de Reflexión Socio Moral: SROM y una Escala de actitudes. Concluyó que existe relación entre la estructura moral y la tendencia hacia el consumo de drogas. Grimaldo (1999) en una investigación con estudiantes de quinto de secundaria de niveles socioeconómico medio y bajo, encontró un buen número de estudiantes de nivel socioeconómico bajo que se ubicaron en el sub estadio 3 comparados a los del nivel socio-económico medio que puntuaron más alto, ubicándose en el sub estadio 4 del Juicio Moral. No encontró diferencias por género.

Por otra parte, a nivel internacional, Di Blassio et.al (2003) se planteó como objetivo investigar los niveles de Juicio Moral en 1,006 adolescentes entre 15 a 16 años pertenecientes a una escuela media superior de Milán diferenciándolos por nivel socioeconómico. Aplicó un cuestionario basado en dos historias a manera de dilemas morales. Encontró diferencias en el desarrollo moral según el nivel socioeconómico de los estudiantes.

De otro lado, en la misma línea de explorar hasta qué punto las condiciones socioculturales podrían estar afectando, no solo los niveles de desarrollo 
psicológico sino incluso los neuropsicológicos, se hizo el acercamiento al estudio de las funciones neuropsicológicas ejecutivas del lóbulo frontal.

Y es que cuando los niños y adolescentes, especialmente los de zonas rurales del interior del país van a la escuela, llevan consigo una serie de dificultades que no les permite formar y adquirir las competencias básicas de lectura comprensiva, escritura y matemáticas, indispensables para insertarse en el mundo moderno. Y ello estaría reflejando un déficit marcado en la formación y desarrollo de funciones neuropsicológicas ejecutivas básicas, tales como la planificación, anticipación, verificación, flexibilidad mental, inhibición de los impulsos y regulación de la conducta, que según diversos estudios, corresponde ejecutar a los lóbulos frontales (Luria, 1979; Ortiz, 2004; Flores y Ostrosky- Solis, 2008).

Flores y Ostrosky (2012), a propósito de la construcción del BANFE, precisaron y definieron las funciones ejecutivas de la siguiente forma:

Planeación: función humana primordial. Anticipación, programación, establecimiento de secuencias para la conducta que apunta a una meta. Corteza pre frontal dorso- lateral: CPFDL.

Flexibilidad mental: capacidad para cambiar de plan y de acciones si no logra el resultado. Además capacidad de inhibir las anteriores respuestas. CPFDL.

Control inhibitorio: en base a esta capacidad la Corteza pre frontal inhibe respuestas impulsivas, decide entre diversas respuestas posibles, inhibe patrones de respuesta cuando no son útiles. COF y CFM.

Memoria de trabajo: es un tipo de memoria que se mantiene activa mientras el hombre está tratando de lograr sus objetivos. Baddeley $(1990,2003)$ la entendía como: administrador central, retén fonológico y registro visoespacial. CPFDL.

Procesamiento riesgo- beneficio: permite tomar buenas decisiones basados en estados afectivos. Participa la corteza órbito- frontal. Damasio (1998). COF y CFM.

Abstracción: Pensamiento abstracto, clasificación lógica. CPFA.

Metacognición: capacidad para monitorear, controlar y regular al resto de procesos cognitivos y ejecutivos. CPFA.

Estudios recientes indican la relación entre nivel socio- económico y desarrollo de las funciones ejecutivas (Arán, 2011), así como el desarrollo distinto de las funciones ejecutivas en contextos bilingues (Martínez y Henao, 2006). 
Bravo (2014), en la revisión hecha sobre los nuevos aportes de las neurociencias, muestra a la luz de diversas investigaciones, la relación que existe entre el desarrollo cerebral y los estímulos lingüísticos, sociales y emocionales que surgen del ambiente escolar, familiar y social.

Bajo estas consideraciones nos pareció que era importante avanzar en el conocimiento de la neuropsicología de los lóbulos frontales y el papel que juegan en las funciones ejecutivas para poder avanzar a una mayor comprensión de cómo es que se organiza el cerebro para la planificación y organización del comportamiento; lo cual relacionado con el conocimiento de la forma en que se desarrolla la reflexión moral en los niños y jóvenes podría brindar luces sobre su conducta moral.

Por tales razones en el presente estudio se buscó establecer si existirían diferencias en las funciones neuropsicológicas ejecutivas y en el juicio moral, en estudiantes universitarios tomando en cuenta su diferente procedencia sociocultural. Se estudió si a esta diversidad social le corresponde una diversa realidad neuropsicológica y psíquica moral.

\section{Objetivos}

Determinar si existen diferencias en los niveles de desarrollo del juicio moral y de las funciones ejecutivas en estudiantes universitarios de Lima, Huancavelica y Ayacucho, teniendo en cuenta su distinta realidad socio- cultural.

\section{Objetivos específicos}

1. Evaluar el nivel de desarrollo del juicio moral en estudiantes universitarios de Lima, Huancavelica y Ayacucho.

2. Evaluar las funciones neuropsicológicas ejecutivas: memoria de trabajo, fluidez verbal, planificación, flexibilidad cognitiva, inhibición y control de interferencia en estudiantes universitarios de Lima, Huancavelica y Ayacucho.

3. Comparar los niveles de rendimiento en funciones neuropsicológicas ejecutivas y el juicio moral en los estudiantes universitarios examinados.

\section{MÉTODO}

El presente es un estudio descriptivo, de tipo causal comparativo en donde diferenciando a los estudiantes universitarios por su procedencia socio- cultural, se trata de ver como se han desarrollado las variables: juicio moral y funciones neuropsicológicas ejecutivas.

Para el examen del Juicio moral se evaluó a 209 estudiantes de tres lugares geográficos: Lima, Huancavelica y Ayacucho. Todos eran alumnos de educación 
superior, en las especialidades de Educación Inicial, Educación Primaria, Educación Secundaria, Educación Física e inglés. Los estudiantes provenían de las siguientes instituciones: 48 alumnos eran de Lima de la Universidad Nacional Mayor de San Marcos, 77 de la Universidad Nacional de Huancavelica y 84 eran alumnos del Instituto Pedagógico Superior "Nuestra Señora de Lourdes" de Ayacucho. La muestra se escogió a criterio, considerando la posibilidad de acceso a dichos estudiantes y el apoyo de las autoridades.

En cuanto al lugar de nacimiento de los participantes en la Universidad Nacional Mayor de San Marcos, el 72\% había nacido en la ciudad de Lima. En el caso del grupo de estudiantes universitarios de Huancavelica, el 94 nació en esa región. Por último, en la muestra de Ayacucho, el 98\% de estudiantes universitarios nació en Ayacucho. Las edades de la muestra estudiada fue entre 19 a 22 años con ligeros intervalos en cada sub grupo.

En relación al género y la especialidad de los alumnos, se puede ver la siguiente tabla:

Tabla 1

Distribución de la muestra en función del género, especialidad en la educación superior y grupo de pertenencia.

\begin{tabular}{llll}
\hline & $\begin{array}{l}\text { Grupo 1 } \\
\text { Lima } \\
\left(\mathrm{N}_{1}=48\right)\end{array}$ & $\begin{array}{l}\text { Grupo 2 Huancavelica } \\
\left(\mathrm{N}_{2}=77\right)\end{array}$ & $\begin{array}{l}\text { Grupo 3 } \\
\text { Ayacucho } \\
\left(\mathrm{N}_{3}=84\right)\end{array}$ \\
\hline $\begin{array}{l}\text { Género } \\
\text { Femenino }\end{array}$ & $\begin{array}{l}4(91.7 \%) \\
\text { Masculino }\end{array}$ & $77(100 \%)$ & $65(79.3 \%)$ \\
Especialidad & $4(8.3 \%)$ & $0(0 \%)$ & $17(20.7 \%)$ \\
Educación inicial & $0(0 \%)$ & $77(100 \%)$ & $37(44.0 \%)$ \\
Educación primaria & $48(100 \%)$ & $0(0 \%)$ & $0(0 \%)$ \\
Educación secundaria & $0(0 \%)$ & $0(0 \%)$ & $13(15.5 \%)$ \\
Educación física & $0(0 \%)$ & $0(0 \%)$ & $21(25.0 \%)$ \\
Inglés & $0(0 \%)$ & $0(0 \%)$ & $13(15.5 \%)$ \\
\hline
\end{tabular}

Respecto a la edad de los estudiantes de la muestra, se encuentran entre los 19 a 22 años con ligeros intervalos en cada sub grupo. Así tenemos que en el caso del primer grupo de estudiantes proveniente de la Universidad Nacional Mayor de San Marcos, su edad media fue de 21.9 (E.E.M. = .21; D.E. = 1.43). En el caso del segundo grupo correspondiente a los estudiantes de Huancavelica, su edad media fue de 21.5 (E.E.M. $=.27$ : D.E. $=2.40$ ).

Finalmente, en el tercer grupo procedente de la región de Ayacucho, su edad media fue de 19.26 (E.E.M. = .12; D.E. = 1.10). En tanto que para examinar las Funciones neuropsicológicas ejecutivas, cuya evaluación necesariamente tenía que ser individual, se evaluó a 23 alumnos de la Universidad de San Marcos, 20 de la Universidad de Huancavelica y 15 alumnos de la Universidad San Cristóbal de 
Huamanga. La población estuvo constituida por alumnos del interior del país, de las zonas sur andinas, Ayacucho y Huancavelica (regiones que sufrieron efectos de la violencia armada años atrás y soportan extrema pobreza) y por los estudiantes de Lima: Universidad Nacional Mayor de San Marcos. Se controlaron las variables: edad, sexo, año de estudios en la universidad, procedencia social y cultural. Al final se trató de observar las diferencias que aparecen entre los grupos a nivel de las variables estudiadas.

\section{Técnicas e instrumentos de recolección de datos:}

Se aplicaron los siguientes instrumentos:

1. Para examinar el Juicio Moral se empleó el Test SROM (test de reflexión socio- moral de Gibbs y Widaman (versión reajustada y abreviada).

2. Batería neuropsicológica de funciones ejecutivas: BANFE, de Flórez y Ostroski ( 2012).

\section{RESULTADOS}

\section{Hipótesis}

Existen diferencias en el nivel de desarrollo de Juicio Moral en los alumnos de educación superior, en función a su realidad socio- cultural.

\section{Variable 1: nivel de Juicio moral}

Se evaluaron los niveles de desarrollo del juicio moral propuesto por Lawrence Kohlberg, un punto de vista cognitivo operatorio que entiende al juicio moral como un proceso intelectual que nos permite razonar y argumentar en base a ciertos valores, ordenarlos de modo jerárquico, lo cual en la vida real nos sirve tomar decisiones y orientar nuestra conducta.

Para la presente investigación se evaluó la moral a través de la aplicación del Cuestionario de Desarrollo Socio Moral de Gibbs \& Widaman (1982), al cual se le hizo algunas modificaciones, abreviándolo y reajustándolo, pero rescatando la estrategia de medir el juicio moral a través de dilemas morales lo cual permitirá clasificar a los sujetos en determinadas etapas del desarrollo moral.

Existen 3 etapas de desarrollo moral: Nivel I Pre convencional; Nivel II Convencional y Nivel III Post Convencional. Cada uno de los niveles, a su vez se compone de dos estadios.

Así el Nivel I Pre convencional que se caracteriza por la ausencia de normas y la moral heterónoma y temor al castigo, tiene estadio 1: Castigo y orientación a la obediencia; y estadio 2: Orientación instrumental. 
El nivel Convencional, donde prevalecen las normas y expectativas de grupo, igualmente tiene dos estadios. Estadio 3: Moral conformista, y estadio 4: Moral de la ley y el orden.

Y el nivel Post convencional, donde predominan los Principios éticos universales y la racionalidad, que se subdivide en Estadio 5: Moral legal, y Estadio 6: Moral de Principios éticos universales.

El dilema y las preguntas que se presentaron a los estudiantes para evaluar los sub estadios de desarrollo moral fueron las siguientes:

Se empleó el dilema kohlbergiano del caso de Juan, quien tenía a su esposa gravemente enferma y que para salvarla necesitaba de una medicina que él no podía comprarla. Al final Juan se enfrenta a un grave dilema: él necesita ayudar a su esposa, para ello piensa que un camino es robar la medicina y salvarle la vida. Lo otro es no robar, porque es un delito, pero su esposa moriría.

1. ¿Qué debe hacer Juan? ¿Deberá robar? ¿No debe robar?

2. ¿Suponiendo que la persona que está muriendo no es la mujer de Juan sino un amigo suyo, quien no tiene a nadie más que ayude? ¿Qué debe hacer Juan? ¿Debe robar? ¿No debe robar?

3. ¿Y si la persona que se está muriendo no el amigo, sino un extraño quien no tiene a nadie más que lo ayude...? ¿Qué debe hacer Juan? ¿Debe robar? ¿No debe robar?

En relación a la primera pregunta, se encontraron los resultados que se presentaran en las tablas y figuras correspondientes.

En el dilema moral de Juan, en la primera pregunta (esposa) se hallaron diferencias significativas en relación al grupo de pertenencia a favor de los estudiantes de Lima.

En la segunda pregunta (amigo) se encontraron diferencias también favorables para el grupo de Lima.

En la tercera pregunta (extraño) también se encontraron diferencias significativas también a favor del grupo de Lima.

En conjunto, se puede observar que los estudiantes del grupo de Lima presentaron mayores niveles de desarrollo del juicio moral en comparación con los estudiantes del grupo 2 (Huancavelica) y del grupo 3 (Ayacucho). Por lo tanto se puede concluir que son los alumnos de Lima, quienes en mayor porcentaje se ubican en el nivel post convencional (nivel más alto del juicio moral) en los 3 aspectos de la moral evaluados. Así, ellos obtienen porcentajes de 31\%, 35\% y 27\% 
respectivamente; a diferencia del grupo de estudiantes de Huancavelica, quienes obtienen porcentajes de 9\%, 5\% y 6.5\%; y los de Ayacucho, 9.5\%, 6\% y $8 \%$ en el mismo nivel post convencional. De otro lado, en el nivel pre- convencional (nivel más bajo del juicio moral), los de Lima obtienen en los tres aspectos examinados, $8 \%, 10 \%$ y $14.7 \%$ respectivamente, mientras que los de Huancavelica obtienen $57 \%, 69 \%$ y $63 \%$; y los de Ayacucho $47 \%, 47 \%$ y $52 \%$ respectivamente.

\section{Tabla 2}

Niveles de juicio moral según región socio-cultural

\begin{tabular}{|c|c|c|c|}
\hline & $\begin{array}{c}\text { Grupo } 1 \\
\text { Lima } \\
\left(N_{1}=48\right)\end{array}$ & $\begin{array}{c}\text { Grupo } 2 \\
\text { Huancavelica } \\
\left(\mathbf{N}_{2}=77\right)\end{array}$ & $\begin{array}{c}\text { Grupo } 3 \\
\text { Ayacucho } \\
\left(N_{3}=84\right)\end{array}$ \\
\hline \multicolumn{4}{|l|}{ Problema Juan -esposa } \\
\hline Estadio 1. Preconvencional & $2(4.2 \%)$ & $9(11.7 \%)$ & $15(17.9 \%)$ \\
\hline Estadio 2. Preconvencional & $2(4.2 \%)$ & $35(45.5 \%)$ & $25(29.8 \%)$ \\
\hline Estadio 3. Convencional & $3(6.3 \%)$ & $7(9.1 \%)$ & $11(13.1 \%)$ \\
\hline Estadio 4. Convencional & $12(25 \%)$ & $9(11.7 \%)$ & $5(6 \%)$ \\
\hline Estadio 5. Postconvencional & $7(14.6 \%)$ & $7(9.1 \%)$ & $7(8.3 \%)$ \\
\hline Estadio 6. Postconvencional & $8(16.7 \%)$ & $0(0 \%)$ & $1(1.2 \%)$ \\
\hline \multicolumn{4}{|l|}{ Problema Juan - Amigo } \\
\hline Estadio 1. Preconvencional & $1(2.1 \%)$ & $15(19.5 \%)$ & $14(16.7 \%)$ \\
\hline Estadio 2. Preconvencional & $4(8.3 \%)$ & $38(49.4 \%)$ & $26(31 \%)$ \\
\hline Estadio 3. Convencional & $7(14.6 \%)$ & $5(6.5 \%)$ & $7(8.3 \%)$ \\
\hline Estadio 4. Convencional & $12(25 \%)$ & $5(6.5 \%)$ & $6(7.1 \%)$ \\
\hline Estadio 5. Postconvencional & $5(10.4 \%)$ & $4(5.2 \%)$ & $5(6 \%)$ \\
\hline Estadio 6. Postconvencional & $12(25 \%)$ & $0(0 \%)$ & $0(0 \%)$ \\
\hline \multicolumn{4}{|l|}{ Problema Juan - Extraño } \\
\hline Estadio 1. Preconvencional & $2(4.2 \%)$ & $21(27.3 \%)$ & $17(20.2 \%)$ \\
\hline Estadio 2. Preconvencional & $6(12.5 \%)$ & $28(36.4 \%)$ & $27(32.1 \%)$ \\
\hline Estadio 3. Convencional & $4(8.3 \%)$ & $4(5.2 \%)$ & $5(6 \%)$ \\
\hline Estadio 4. Convencional & $11(22.9 \%)$ & $3(3.9 \%)$ & $4(4.8 \%)$ \\
\hline Estadio 5. Postconvencional & $1(2.1 \%)$ & $5(6.5 \%)$ & $6(7.1 \%)$ \\
\hline Estadio 6. Postconvencional & $12(25 \%)$ & $0(0 \%)$ & $1(1.2 \%)$ \\
\hline
\end{tabular}

\section{Variable 2: Funciones neuropsicológicas ejecutivas}

\section{Descripción de los participantes}

- Grupo 1. Estuvo constituido por 20 estudiantes de una universidad nacional de Huancavelica, de sexo femenino, cuyas edades fluctúan entre 17 y 24 años de edad, con una edad media de 19.8 (D.E. $=2.04$, E.E.M.=.456).

- Grupo 2. Estuvo constituido por 15 estudiantes de una universidad nacional de Ayacucho, 13 mujeres y 2 varones, cuyas edades fluctúan 
entre 18 y 23 años de edad, con una edad media de 20.47 (D.E. $=1.45$, E.E.M. $=.376)$.

- Grupo 3. Estuvo constituido por 23 estudiantes de una universidad nacional de Lima, 21 del sexo femenino y 2 del sexo masculino, cuyas edades fluctúan entre 21 y 23 años de edad, con una edad media de 21.61 (D.E.=.89, E.E.M.= .196).

\section{Resultados descriptivos: Funciones ejecutivas}

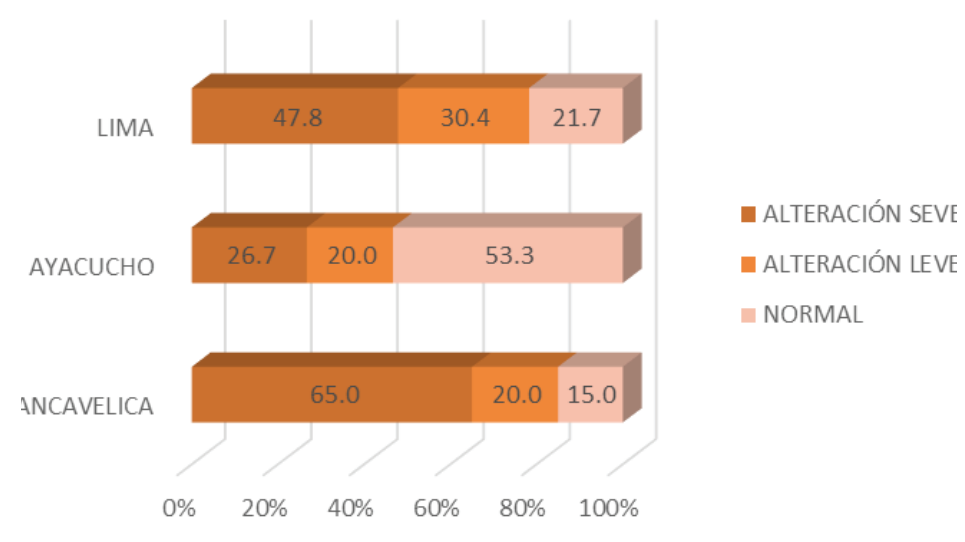

Figura 1. Desempeño de los estudiantes en funciones ejecutivas del área orbitomedial.

NOTA: aunque el autor de la prueba BANFE, utiliza el término ALTERACIÓN para aludir a la categorización producto de los bajos puntajes alcanzados por el sujeto, podemos entender dicho término como sinónimo de DEFICIENCIAS.

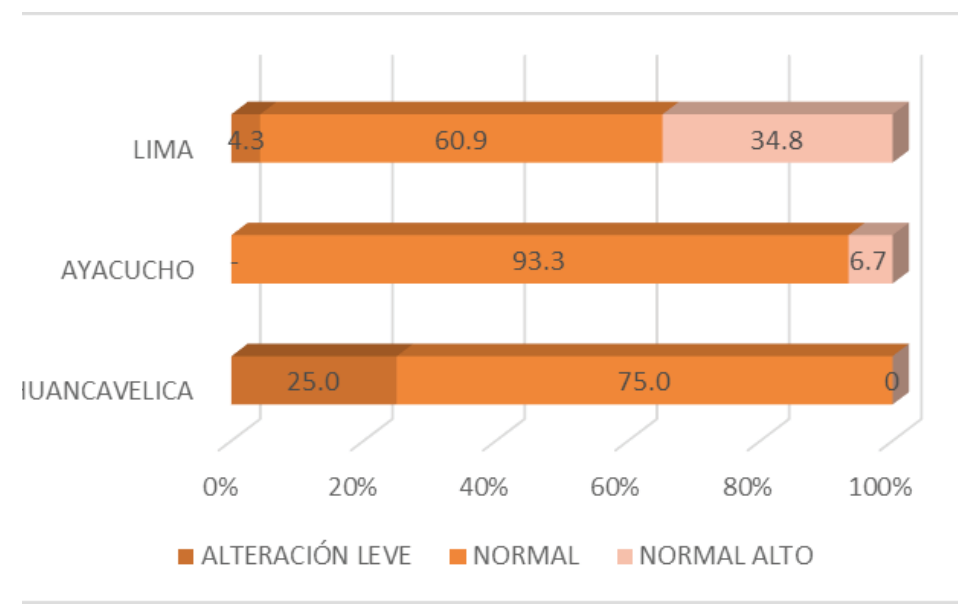

Figura 2. Desempeño de los estudiantes en funciones ejecutivas de áreas pre frontal anterior. 


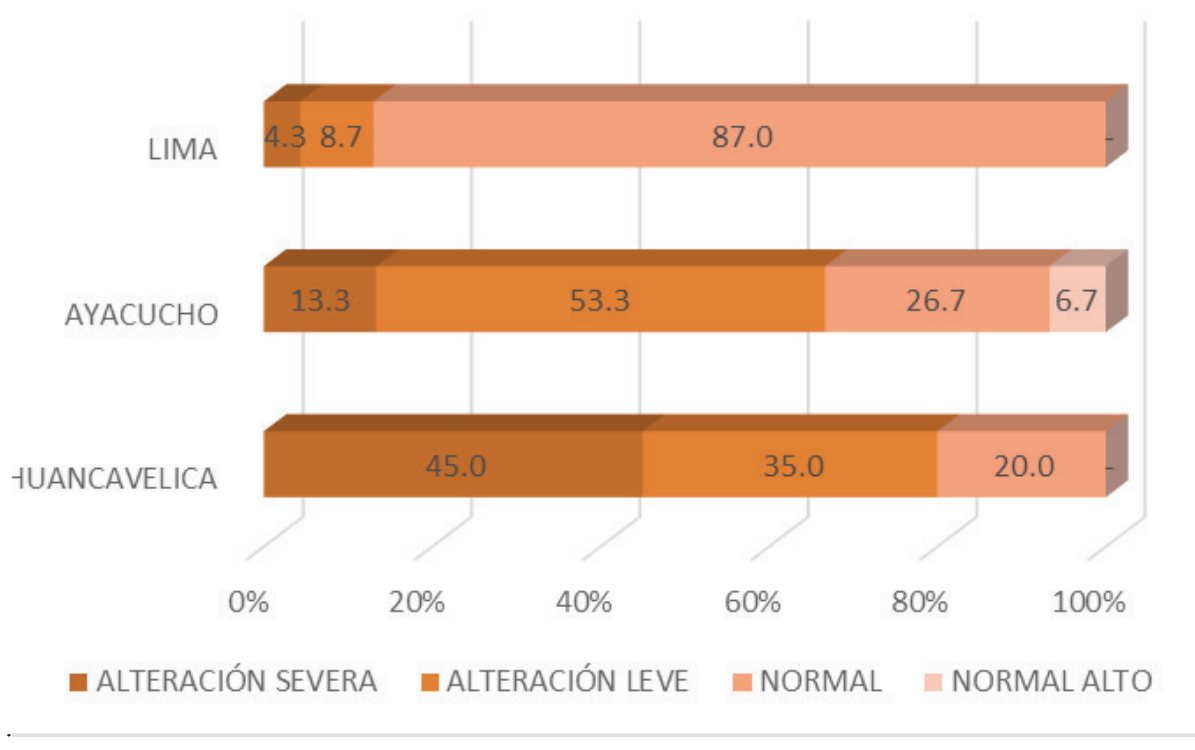

Figura 3. Desempeño de los estudiantes en funciones ejecutivas de las áreas dorso-laterales

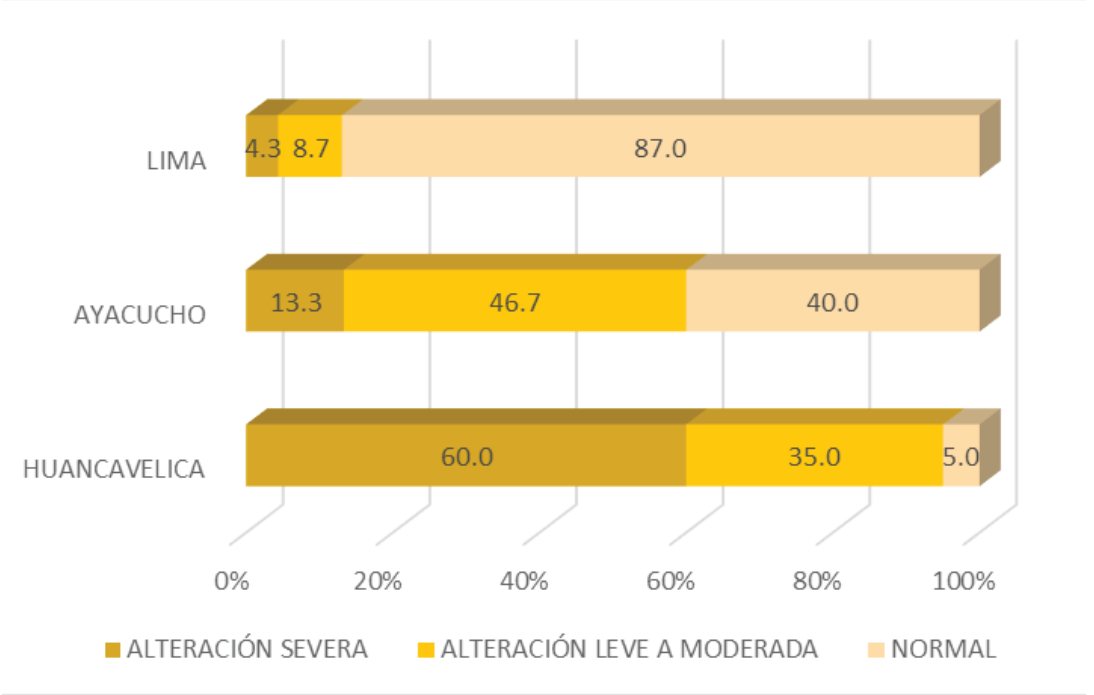

Figura 4. Desempeño global de los estudiantes universitarios en las funciones ejecutivas. 


\section{Comparación de grupos}

\section{Tabla 3}

Comparación de grupos según los resultados en las pruebas de funciones ejecutivas de las áreas orbito medial.

\begin{tabular}{|c|c|c|c|c|c|c|c|c|c|c|}
\hline & \multicolumn{5}{|c|}{ Puntuaciones naturales } & \multicolumn{5}{|c|}{ Puntuaciones normalizadas } \\
\hline & Media & $\mathrm{DE}^{\mathrm{a}}$ & $\begin{array}{l}\text { Rango } \\
\text { promedio }\end{array}$ & $\chi^{2 \mathrm{~b}}$ & $\mathrm{p}^{\mathrm{b}}$ & Media & $\mathrm{DE}^{\mathrm{a}}$ & $\begin{array}{l}\text { Rango } \\
\text { promedio }\end{array}$ & $\chi^{2 b}$ & $\mathrm{p}^{\mathrm{b}}$ \\
\hline Huancavelica & 183 & 6.21 & 23.08 & 5.530 & .063 & 64.3 & 16.59 & 22.88 & 5.715 & .057 \\
\hline Ayacucho & 188.46 & 7.42 & 36.43 & & & 79.8 & 19.83 & 36.37 & & \\
\hline Lima & 186.17 & 7.07 & 30.57 & & & 73.3 & 18.76 & 30.78 & & \\
\hline
\end{tabular}

${ }^{a}$ DE: Desviación estándar

begún la prueba de Kruskal-Wallis (grados de libertad: 2)

De acuerdo a la tabla 3, no se encontraron diferencias entre los desempeños en las tareas asociadas a las áreas orbitofrontales de los grupos de estudiantes universitarios de diferentes realidades socioculturales.

\section{Tabla 4}

Comparación de grupos según los resultados en las pruebas de funciones ejecutivas de las áreas pre frontales anterior

\begin{tabular}{|c|c|c|c|c|c|c|c|c|c|c|}
\hline & \multicolumn{5}{|c|}{ Puntuaciones naturales } & \multicolumn{5}{|c|}{ Puntuaciones normalizadas } \\
\hline & Media & $\mathrm{DE}^{\mathrm{a}}$ & $\begin{array}{l}\text { Rango } \\
\text { promedio }\end{array}$ & $\chi^{2 b}$ & $\mathrm{p}^{\mathrm{b}}$ & Media & $\mathrm{DE}^{\mathrm{a}}$ & $\begin{array}{l}\text { Rango } \\
\text { promedio }\end{array}$ & $\chi^{2 b}$ & $\mathrm{p}^{\mathrm{b}}$ \\
\hline Huancavelica & 18.25 & 2.05 & 18.75 & 14.635 & .001 & 91.35 & 10.98 & 18.75 & 14.635 & .001 \\
\hline Ayacucho & 19.93 & 1.49 & 30.33 & & & 100.33 & 8.01 & 30.33 & & \\
\hline Lima & 21.01 & 2.37 & 38.30 & & & 106.48 & 12.71 & 38.30 & & \\
\hline
\end{tabular}

a DE: Desviación estándar

b Según la prueba de Kruskal-Wallis (grados de libertad: 2)

De acuerdo a la tabla 4, se encontraron diferencias significativas entre los desempeños en las tareas asociadas al área pre frontal anterior de los grupos de estudiantes universitarios de diferentes realidades socioculturales. Al hacer un análisis de pares, no paramétrico, con el estadístico de Mann Whitney se encontró que las diferencias se presentan entre: los estudiantes de Huancavelica y Ayacucho $(\mathrm{U}=79.5, \mathrm{p}=.01, \mathrm{r}=.40)$; $\mathrm{y}$ entre los estudiantes de Huancavelica y Lima $(\mathrm{U}=$ $85.50, \mathrm{p}=.034, \mathrm{r}=.54)$; pero no se encuentran diferencias significativas entre los estudiantes de Ayacucho y Lima $(\mathrm{U}=114.50, \mathrm{p}=.083)$. Son los estudiantes de Lima, los que alcanzan las mayores puntuaciones; mientras que los estudiantes de Huancavelica obtienen los menores puntajes. 
Tabla 5

Comparación de grupos según los resultados en las pruebas de funciones ejecutivas de las áreas dorso laterales

\begin{tabular}{|c|c|c|c|c|c|c|c|c|c|c|}
\hline & \multicolumn{5}{|c|}{ Memoria de trabajo } & \multicolumn{5}{|c|}{ Funciones ejecutivas } \\
\hline & Media & $\mathrm{DE}^{\mathrm{a}}$ & $\begin{array}{c}\text { Rango } \\
\text { promedio }\end{array}$ & $\mathrm{X}^{2 \mathrm{~b}}$ & $\mathrm{p}^{\mathrm{b}}$ & Media & $\mathrm{DE}^{\mathrm{a}}$ & $\begin{array}{c}\text { Rango } \\
\text { promedio }\end{array}$ & $\mathrm{X}^{2 \mathrm{~b}}$ & $\mathrm{p}^{\mathrm{b}}$ \\
\hline Huancavelica & 98.35 & 12.72 & 23,30 & 4.137 & .126 & 86.45 & 12.10 & 15,88 & 29.240 & .000 \\
\hline Ayacucho & 103.47 & 10.51 & 33,07 & & & 97.40 & 13.05 & 26,30 & & \\
\hline \multirow[t]{2}{*}{ Lima } & 104.74 & 60.75 & 32,57 & & & 102.34 & 60.16 & 43,43 & & \\
\hline & \multicolumn{5}{|c|}{ Dorso lateral - Puntuaciones naturales } & \multicolumn{5}{|c|}{ Dorso lateral - Puntuaciones normalizadas } \\
\hline Huancavelica & 184.45 & 19.14 & 15.90 & 29.089 & .000 & 71.70 & 14.62 & 16.45 & 26.283 & .000 \\
\hline Ayacucho & 200.87 & 15.68 & 26.33 & & & 83.87 & 12.12 & 26.77 & & \\
\hline Lima & 207.09 & 60.75 & 43.39 & & & 96.44 & 11.88 & 42.63 & & \\
\hline
\end{tabular}

a DE: Desviación estándar

b Según la prueba de Kruskal-Wallis (grados de libertad: 2)

De acuerdo a la tabla 5, no se encontraron diferencias significativas entre los resultados obtenidos en las tareas de memoria de trabajo de los grupos de estudiantes universitarios de diferentes realidades socioculturales. Sin embargo, las diferencias son significativas entre los desempeño en los sub tests asociados a las funciones ejecutivas del área dorso-lateral. Al hacer un análisis de pares, no paramétrico, con el estadístico de Mann Whitney se encontró que existen diferencias entre: los estudiantes de Huancavelica y Ayacucho $(U=75.0, p=.012, r=.42)$; entre los estudiantes de Huancavelica y Lima $(\mathrm{U}=32.5, \mathrm{p}=.000, \mathrm{r}=.73)$; y entre los estudiantes de Ayacucho y Lima $(\mathrm{U}=49.5, \mathrm{p}=.000, \mathrm{r}=.59)$. Los desempeños de los estudiantes de Lima son significativamente mayores a los de sus pares de Ayacucho y Huancavelica. Estos resultados son similares a los encontrados en el análisis comparativo de las puntuaciones totales de los subtests que evalúan las funciones de las áreas dorso laterales. Al hacer un análisis de pares, no paramétrico, con el estadístico de Mann Whitney se encontró que las diferencias se presentan entre: los estudiantes de Huancavelica y Ayacucho $(\mathrm{U}=75.5, \mathrm{p}=.013, \mathrm{r}=.42)$; entre los estudiantes de Huancavelica y Lima $(\mathrm{U}=32.5, \mathrm{p}=.000, \mathrm{r}=.73)$; y entre los estudiantes de Ayacucho y Lima $(\mathrm{U}=50.5, \mathrm{p}=.000, \mathrm{r}=.59)$.

Tabla 6

Comparación de grupos según los resultados en las pruebas de funciones ejecutivas.

\begin{tabular}{|c|c|c|c|c|c|c|c|c|c|c|}
\hline & \multicolumn{5}{|c|}{ Puntuaciones naturales } & \multicolumn{5}{|c|}{ Puntuaciones normalizadas } \\
\hline & Media & $\mathrm{DE}^{\mathrm{a}}$ & $\begin{array}{c}\text { Rango } \\
\text { promedio }\end{array}$ & $\mathrm{X}^{2 \mathrm{~b}}$ & $\mathrm{p}^{\mathrm{b}}$ & Media & $\mathrm{DE}^{\mathrm{a}}$ & $\begin{array}{c}\text { Rango } \\
\text { promedio }\end{array}$ & $\mathrm{X}^{2 \mathrm{~b}}$ & $\mathrm{p}^{\mathrm{b}}$ \\
\hline Huancavelica & 385.70 & 20.86 & 15.03 & 27.470 & .000 & 68.40 & 12.40 & 15.03 & 27.515 & .000 \\
\hline Ayacucho & 409.27 & 18.78 & 29.53 & & & 82.73 & 11.32 & 29.50 & & \\
\hline Lima & 424.52 & 17.88 & 42.07 & & & 92.00 & 10.74 & 42.09 & & \\
\hline
\end{tabular}

a DE: Desviación estándar

b Según la prueba de Kruskal-Wallis (grados de libertad: 2) 
De acuerdo a la tabla 6, se encontraron diferencias significativas entre los rendimientos en puntuaciones totales de las tareas de funciones ejecutivas de los grupos de estudiantes universitarios de diferentes realidades socioculturales. Al hacer un análisis de pares, no paramétrico, con el estadístico de Mann Whitney se encontró que las diferencias se presentan entre: los estudiantes de Huancavelica y Ayacucho $(\mathrm{U}=55.00, \mathrm{p}=.002, \mathrm{r}=.54)$; entre los estudiantes de Huancavelica y Lima $(\mathrm{U}=35.50, \mathrm{p}=.000, \mathrm{r}=.72)$; y entre los estudiantes de Ayacucho y Lima (U $=78.00, \mathrm{p}=.004, \mathrm{r}=.46)$. Al igual que en las áreas anteriormente descritas, son los estudiantes de Lima quienes alcanzan las mayores puntuaciones, seguidos de los estudiantes de Ayacucho, siendo los estudiantes de Huancavelica quienes obtienen los menores puntajes.

\section{DISCUSION}

Los resultados principales del presente estudio muestran las importantes diferencias que aparecen entre los grupos, en primer lugar, del desarrollo del juicio moral, a favor del grupo de Lima. Llama la atención esa especie de contraste entre lo que mayoritariamente rinden los alumnos de Huancavelica, ubicándose en su gran mayoría, en los 3 aspectos del dilema moral estudiado, en el nivel preconvencional, y los de Lima, quienes mayoritariamente se ubican en el nivel post convencional.

Ello nos lleva a pensar hasta qué punto tiene aún preeminencia en el juicio moral de los alumnos de la universidad del interior, lo que Piaget (1971) llamaba la moral Heterónoma, es decir aquella orientación de la conducta moral en función a la presencia de la autoridad externa y el temor al castigo, no habiéndose estructurado aun adecuadamente la moral que toma en cuenta la ley, lo social, y más adelante los valores éticos universales.

En relación a dicha moral heterónoma, habría que tomar en consideración las particulares condiciones sociales, históricas, culturales y familiares, en las que han crecido dichos jóvenes: autoritarismo, violencia y dominación. Y este aspecto tiene que ver mucho con el tipo de educación que reciben desde los primeros años, y con el estilo de crianza al interior de las familias.

Este es un punto importante a tomar en cuenta, cuando de analizar las causas de la violencia en el Perú, especialmente en las zonas de Ayacucho y Huancavelica, se trata.

Por lo demás en la moral heterónoma, prima el interés personal, un cierto hedonismo, y una visión muy concreta y empírica de las cuestiones sociales y morales. Precisamente cuando Kohlberg (1995), habla de moral pre convencional, quiere referirse a ese tipo de juicio moral, en donde aún no impera la norma, ni la ley, muy característico de las sociedades anómicas, en donde encontramos el caldo de cultivo para la corrupción, así como para la violencia. 
Todo ello, nos estaría indicando que se hace necesario reforzar el trabajo educativo en el campo de los valores y del juicio moral. Se evidencia además, que llegar al nivel universitario, no es garantía de avanzar en el desarrollo del juicio moral. Todo depende del tipo de educación que se reciba en los diferentes niveles, del entorno familiar, de las experiencias sociales que atraviese el individuo, de sus grados de reflexión y avances en sus niveles de conciencia.

De otro lado, en relación a las funciones neuropsicológicas ejecutivas (Flores y Ostrosky, 2012), apreciamos que también aparecen diferencias significativas entre los grupos, en este caso a favor del grupo de Lima.

Hoy se sabe que las funciones ejecutivas, que dependen del buen funcionamiento de los lóbulos frontales, se encargan de la planeación, anticipación, regulación y verificación de la conducta (Luria, 1979), así como de la formulación de intenciones y programas de acción. Este conjunto de funciones psíquicas son las más complejas del ser humano (evolutivamente también son las que aparecen más tardíamente), y su alteración afecta los sistemas motivacionales y volitivos del hombre, así como su conducta social.

En la actualidad se estudia a las funciones ejecutivas en términos de la capacidad de organización de la información de la que se nutre la persona, el control inhibitorio importante para regular y controlar las conductas impulsivas, la flexibilidad mental que nos permite cambiar la estrategia de acción cuando aquella no está dando resultado, la producción de hipótesis, la planeación cognitiva, la capacidad de abstracción, la memoria de trabajo, y la metacognición.

A nivel neuropsicológico las estructuras estudiadas que son básicas para las funciones ejecutivas son: la corteza orbito- frontal: cuya función principal es la regulación de los estados emocionales- afectivos, la toma de decisiones (Damasio, 1998) y la detección de cambios en el ambiente en función al cálculo de riesgo/ beneficio.

El área pre frontal medial, participa en los procesos atencionales, en los procesos de detección y solución de problemas, y en el control inhibitorio. Asimismo los niveles más superiores participan en los procesos de empatía y de autoconocimiento (teoría de la mente).

Las áreas dorso-laterales intervienen directamente en los procesos de planeación, en la memoria de trabajo, en la fluidez mental y verbal, en la producción de hipótesis; también los niveles más superiores, en el monitoreo, ajuste y metacognición de la conducta. Todo ello es base para organizar mejor la actividad social de la persona, así como para la integración de la conciencia.

Teniendo en cuenta todo ello, habría que interpretar nuestros resultados del modo siguiente: en lo que se refiere al funcionamiento ejecutivo que depende de 
áreas orbito- frontales, no se halló diferencias entre los grupos. Ello nos estaría indicando que en lo que corresponde a la regulación de los estados emocionalesafectivos y la toma de decisiones los alumnos universitarios rinden igual, pero ello no significa que se encuentren funcionando a un nivel adecuado, sino que los 3 grupos han rendido muy bajo. Ante tareas de flexibilidad mental, como pasar a un nuevo criterio o estrategia, cuando se les señala que no está bien lo que están haciendo, ellos siguen con el criterio anterior. O para diferenciar mentalmente dos tipos de información que se solapan, y actuar de acuerdo a lo que corresponde (como en pruebas de Stroop), igualmente tienen muchas deficiencias.

En donde se aprecian con claridad las diferencias es en lo que corresponde a las funciones ejecutivas que dependen del área prefrontal anterior y en las áreas dorsolaterales, a favor del grupo de Lima. En particular en lo que se refiere a las áreas dorsolaterales, que serían las de más reciente aparición y que son exclusivas de la organización neocortical del cerebro humano, examinadas a través de tareas de flexibilidad mental, fluidez verbal, productividad, planeación viso- espacial y secuencial, control de codificación y memoria de trabajo, se puede apreciar que los alumnos de Lima aparecen con rendimientos cualitativamente superiores a los de sus semejantes de la universidades del interior.

Igualmente cuando se comparan los rendimientos globales en las funciones ejecutivas, también los alumnos de Lima, muestran los mejores puntajes.

Desde un punto de vista cualitativo, debemos decir que tal como se muestra en los resultados descriptivos, un buen grupo de alumnos de provincias, mostrarían alteraciones o deficiencias de leve a moderadas (un buen grupo de Huancavelica, incluso muestra deficiencia severa) en las funciones ejecutivas.

Aquí la explicación tentativa, sería la de tomar en cuenta las condiciones sociales, económicas, culturales en las que han crecido dichos jóvenes. Es conocida la influencia negativa que ejercen los factores disortogénicos en el desarrollo psicológico de nuestros niños y adolescentes. Gonzáles (2006) ha remarcado las perturbaciones cognitivas, lingüísticas y afectivas, que producen las condiciones de pobreza extrema, la desnutrición, la marginación social, el analfabetismo de los padres, el problema lingüístico (presencia de lenguas originarias: quechua, aymara o lengua amazónica como lengua materna), en dichos niños y jóvenes.

Habría que preguntarnos hasta qué punto todo ello, aparte de influir negativamente en la conformación y desarrollo de las funciones psíquicas, también está actuando a nivel de la conformación de las redes neuronales más complejas del hombre: las funciones ejecutivas del lóbulo frontal. 


\section{CONCLUSIONES}

1. Se hallaron diferencias significativas en el conjunto de las puntuaciones que examinaban Juicio moral a favor del grupo de estudiantes de Lima, quienes en buenos porcentajes alcanzan el nivel de moral post convencional o de principios éticos, mientras que los estudiantes de Huancavelica alcanzan en su mayoría los niveles más deficitarios: el nivel pre- convencional; es decir una moral básicamente heterónoma.

2. Igualmente en lo que concierne a las funciones neuropsicológicas ejecutivas aparecen diferencias significativas a favor el grupo de Lima, particularmente en lo referente a las funciones neuropsicológicas de las áreas pre frontal anterior y dorso- lateral del lóbulo frontal; así como en los puntajes totales de las funciones ejecutivas. Respecto a esta variable se aprecia que la mayor cantidad de estudiantes con deficiencias o alteraciones de las funciones ejecutivas (niveles leve, moderado y severo), pertenecen a la Universidad de Huancavelica.

3. Los instrumentos (pruebas, técnicas y Batería) diseñados y utilizados en el examen de las variables mencionadas, mostraron su valor y utilidad.

4. Se discute la importancia de los factores socio- culturales, los llamados factores disortogénicos (González, 2006): pobreza extrema, la desnutrición, la marginación social, el analfabetismo de los padres, el problema lingüístico (presencia de lenguas originarias: quechua, aymara y lenguas amazónicas como lengua materna), en el desarrollo psicológico y neuropsicológico de los niños y jóvenes de nuestro país.

\section{REFERENCIAS}

Bravo, L. (2014). Psicología cognitiva y neurociencias de la educación en el aprendizaje del lenguaje escrito y las matemáticas. Lima. Revista de investigación en psicología. Vol. 17. $\mathrm{N}^{\circ} 2$.

Canales, R. (1991). Juicio moral y actitudes ante las drogas en adolescentes de sectores populares de Lima Metropolitana. Tesis de grado en Psicología. Lima. Universidad Nacional Mayor de San Marcos.

Canales, R. (2014). Variaciones dialectales del castellano y distancias comunicacionales en estudiantes universitarios iniciales de educación. Un estudio sobre habilidades psicolingüisticas y bilingüismo en el Perú. Lima. Instituto de Investigaciones Psicológicas. UNMSM.

Flores, J. y Ostrosky, F. (2008). Batería de funciones ejecutivas, presentación. Revista de neuropsicología, neuropsiquatría y neurociencias. 8 (1).

Flores, J. y Ostrosky, F. (2012). Desarrollo neuropsicológico de lóbulos frontales y funciones ejecutivas. México. Manual Moderno. 
Gibbs, J. \& Widaman, K. (1982). Social Intelligence. Measuring Development of sociomoral reflection. USA. Prentice Hall International.

Gonzalez, Raúl (2006). Problemas psicolinguísticos en el Perú. Lima. Perú. Edic. Norma Reátegui.

Grimaldo, M. (1999). ¿Hacia dónde van nuestros Jóvenes? Un estudio del juicio moral en estudiantes de nivel socioeconómico medio y bajo. Lima: Universidad de San Martín de Porres.

Grimaldo, M. (2002). Adaptación del Cuestionario de Reflexión Socio Moral (SROM) de Gibbs \& Widaman. Lima: Universidad de San Martín de Porres.

Kuhmerker, L. (1995). L' Ereditá di Kohlberg. Intervento educativo e clínico. Firenze. Giunti Gruppo Editoriale.

Luria, A. (1979). El cerebro en acción. Barcelona. Edit. Fontanella.

Martínez, M. y Henao, G. Desempeño en la función ejecutiva de niños expuestos a ambientes de enseñanza bilingües. Medellín. Revista de investigación psicoeducativa $\mathrm{N}^{\circ} 10$.

Montenegro, C.E. (1987). Un estudio sobre el nivel de Juicio moral y el nivel de pensamiento en un grupo de maestros de educación infantil. Tesis de Maestría en Psicología. Lima. Universidad Peruana Cayetano Heredia.

Neyra, H. (1997) Hacia la tercera mitad. Ensayos de relectura herética. Lima. SIDEA.

Piaget, J. (1971). El criterio moral en el niño. Barcelona: Fontanella.

Ortiz, P. (2004). Introducción a la psicobiología del hombre. Lima. Fondo editorial UNMSM.

Vargas Llosa, M. (2012). La civilización del espectáculo. Lima. Alfaguara. 